\title{
Management of unstable angina based on considerations of aetiology
}

\author{
E Braunwald
}

Unstable angina is not a specific disease but a clinical syndrome, more akin to hypertension than to streptococcal endocarditis. The classification of unstable angina has, until now, been based on simple clinical descriptors, such as whether the ischaemic pain is on exertion and accelerating, or whether it occurs at rest, ${ }^{1}$ and whether or not ischaemia persists despite vigorous anti-ischaemic treatment. The ECG, particularly the presence or absence of changes in the ST segment, has also been useful as a classification tool. In addition, markers of myocardial damage, such as the cardiac specific troponins $\mathrm{T}$ or I are being used increasingly in the classification of these patients. ${ }^{2}$

Such clinical and laboratory descriptors are valuable in predicting prognosis, but they provide little or no information about the aetiology of unstable angina. Such information is important because it allows specific rather than empiric management. To return to the analogy with hypertension, the identification of specific subtypes such as renovascular hypertension or mineralocorticoid induced hypertension has allowed specific and more effective treatment to be developed. Similar advances may be anticipated in unstable angina.

The work of Maseri has been fundamental in the development of our thinking about the cause of unstable angina. ${ }^{3}$ From an aetiological perspective, unstable angina may be classified as thrombosis, severe progressive arterial obstruction, coronary vasospasm/vasoconstriction, inflammation and increased myocardial oxygen consumption. ${ }^{4}$

\section{Aetiological mechanisms}

TYPE I: THROMBOSIS

A non-occlusive thrombus at the site of a fissured or ruptured atherosclerotic plaque is the most frequent pathogenic mechanism in unstable angina. Rational treatment should therefore be aimed directly at the thrombus, by means of antithrombotic agents. These have consisted of unfractionated heparin and aspirin. The recent results of the $\operatorname{ESSENCE}^{5}$ and TIMI $11 \mathrm{~B}^{6}$ trials provide evidence that the low molecular weight heparin (LMWH) enoxaparin may be more effective than unfractionated heparin in unstable angina and is potentially of great value in the management of these patients.

Considerable interest has been expressed in new antiplatelet agents, such as glycoprotein (GP) IIb/IIIa receptor inhibitors, as a means of halting the thrombotic process. Most of the early trial evidence has focused on the use of these agents as adjuncts to percutaneous coronary intervention, ${ }^{78}$ but they have also been shown to be effective in patients with unstable angina who have not undergone percutaneous coronary intervention. ${ }^{9}$ Theoretically, LMWHs and GP IIb/IIIa receptor inhibitors are likely to demonstrate synergistic actions, although trials of combination treatment with these agents have not been completed yet.

TYPE II: SEVERE PROGRESSIVE ARTERIAL OBSTRUCTION

Until about 10 years ago, progressive arterial obstruction was thought to be the most common cause of unstable angina. Although there is now evidence that non-occlusive thrombosis is more common, accelerated atherosclerosis remains an important aetiology. ${ }^{10}$ The cause of this acceleration is not yet clear; the occurrence of subclinical, frequent minor thrombi has been suggested as a possible mechanism. If this mechanism is confirmed, prolonged antiplatelet treatment with an oral GP IIb/IIIa receptor inhibitor could be highly effective. Trials of oral GP IIb/IIIa receptor inhibitors are currently ongoing, and may establish a place for long term GP IIb/IIIa receptor inhibition in this condition.

Restenosis after initially successful percutaneous coronary intervention or coronary artery bypass grafting may also cause progressive mechanical obstruction and lead to unstable angina. In such cases, the most appropriate management is repeat revascularisation.

TYPE III: CORONARY VASOSPASM/

VASOCONSTRICTION

Dynamic constriction of the coronary arteries may affect either the large conductance vessels

\section{Trial acronyms}

CAPTURE: C7e3 Fab AntiPlatelet Therapy in Unstable REfractory angina

EPILOG: Evaluation of Percutaneous transluminal coronary angioplasty to Improve Long term Outcome of cF7E3 Glycoprotein receptor blockade

ESSENCE: Efficacy and Safety of Subcutaneous Enoxaparin in Non-Q wave Coronary Events

PRISM-PLUS: Platelet Receptor Inhibition for ischaemic Syndrome Management in Patients Limited by Unstable Signs and symptoms

TIMI: Thrombolysis In Myocardial Infarction 


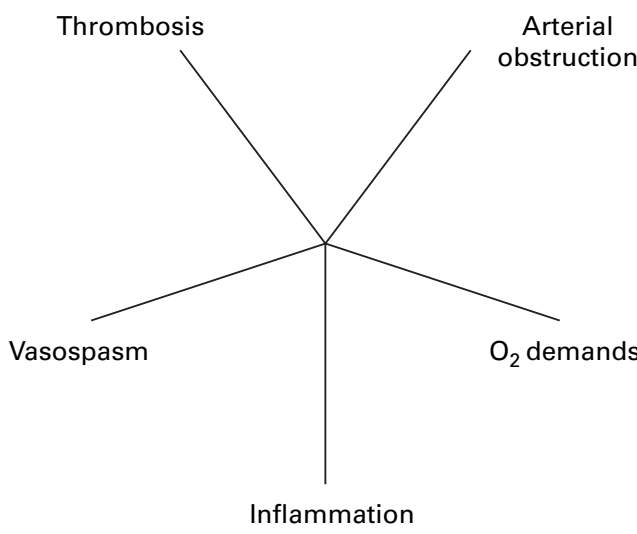

Figure 1 Aetiology of unstable angina. Modified from Braunwald E. Circulation 1998;98:2219-22.5

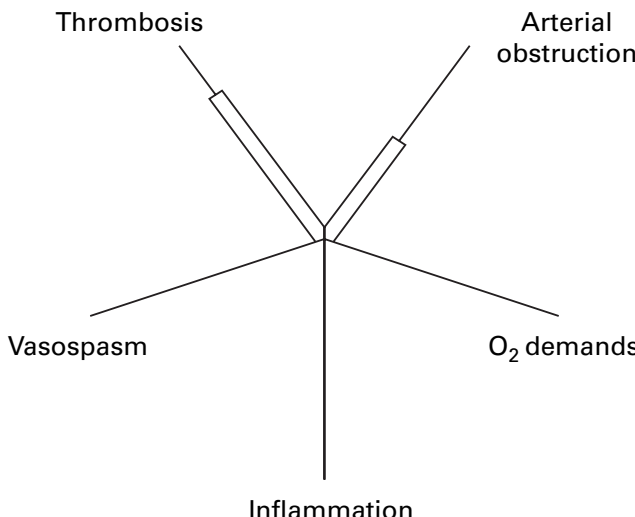

Figure 2 Subtotal thrombotic occlusion on a background of moderate arterial obstruction: a common cause of unstable angina. Modified from Braunwald E. Circulation 1998;98:2219-22.5

as in Prinzmetal's angina, or the small coronary resistance arteries as in microvascular angina. The most effective treatment consists of coronary artery vasodilators, nitrates, and calcium channel blockers.
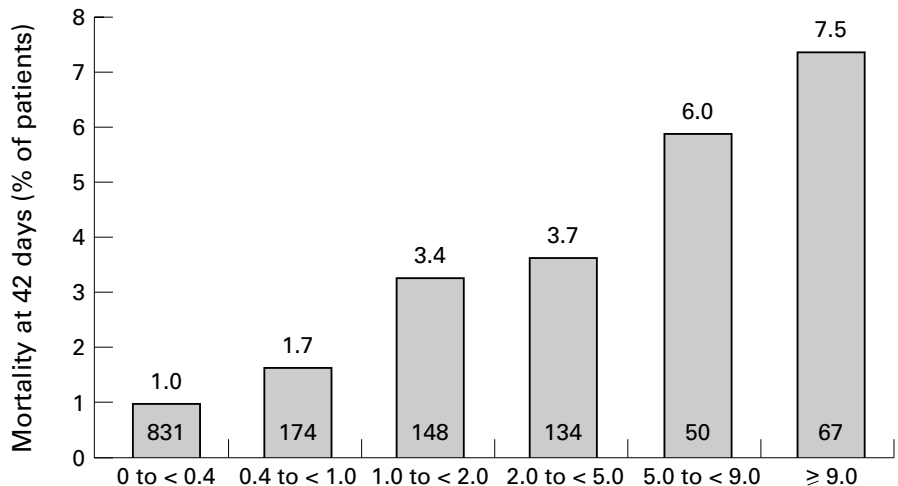

Cardiac troponin I (ng/ml)

$\begin{array}{lcccccc}\text { Risk ratio } & 1.0 & 1.8 & 3.5 & 3.9 & 6.2 & 7.8 \\ 95 \% \text { confidence } & - & 0.5-6.7 & 1.2-10.6 & 1.3-11.7 & 1.7-22.3 & 2.6-23.0\end{array}$
interval

Figure 3 Mortality rates at 42 days (without adjustment for baseline characteristics) according to ranges of cardiac troponin I concentrations measured at enrolment (baseline). The numbers in the bars are the number of patients within each range of troponin I concentrations (the percentage of patients in each range are shown above the bars).

$p=0.001$ for the increase in the mortality rate (and the risk ratio for mortality) with increasing concentrations of cardiac troponin I at enrolment. Reproduced from Antman et $a l^{2}$ with permission of the Massachusetts Medical Society.
TYPE IV: INFLAMMATION

There is growing evidence that unstable angina may be caused by inflammation of the atherosclerotic plaque. Increased activity of macrophages in plaques can cause the release of proteinases such as collagenase and stromelysin, which can break down the extracellular matrix and lead to plaque rupture. The inflammatory response is reflected in raised serum concentrations of markers such as $\mathrm{C}$ reactive protein (CRP) and serum amyloid $\mathrm{A}$; these offer convenient, although indirect, measures of the degree of cytokine activation, which in turn relates to macrophage activity. Many patients with unstable angina have serum CRP concentrations above the upper limit of normal of $3 \mathrm{mg} / \mathrm{l}$, and both the short term and the long term prognosis is poorer in such patients. ${ }^{11} 12$

There is mounting epidemiological evidence that plaque instability may be triggered by infection with organisms such as Chlamydia pneumoniae, Helicobacter pylori, cytomegalovirus or some other as yet unidentified microorganism. C pneumoniae has also been detected in atherosclerotic lesions. ${ }^{13}$ In pilot studies, treatment with macrolide antibiotics has been shown to improve the prognosis in patients following acute coronary syndromes. ${ }^{14}{ }^{15}$ Should an infective aetiology be confirmed in a subgroup of patients, the macrolide antibiotics would offer specific, inexpensive, and relatively safe treatment.

TYPE V: INCREASED MYOCARDIAL OXYGEN CONSUMPTION

When myocardial oxygen supply is compromised by a flow limiting atherosclerotic plaque, an increase in myocardial oxygen demand may be sufficient to trigger unstable angina. Patients with chronic stable angina may develop unstable angina with thyrotoxicosis, infection, fever, or tachyarrhythmias, all of which increase myocardial oxygen demand. Treatment should be directed at managing the underlying illness and using $\beta$ blockers to control the heart rate and reduce myocardial oxygen consumption.

Figure 1 illustrates a model that may be used to represent the balance of aetiologies and provide a guide to treatment. For an individual patient, the diagram could be marked to show the relative importance of each of the five causal factors. The most common presentation is a patient with moderate arterial obstruction who develops a subtotal thrombotic occlusion (fig 2). These are the patients who are most likely to benefit from both antithrombotic and antiplatelet treatment. In other patients, identification of the dominant cause will require quite different management such as revascularisation for the patients with severe mechanical obstruction, or vasodilator treatment when vasospasm is the predominant cause. In many patients, more than one causal factor is present.

\section{Clinical use of biochemical markers}

Data from the TIMI IIIB trial provides an example of how the information gained from measurement of clinical markers can be put to practical use in predicting prognosis. ${ }^{2}$ The risk 


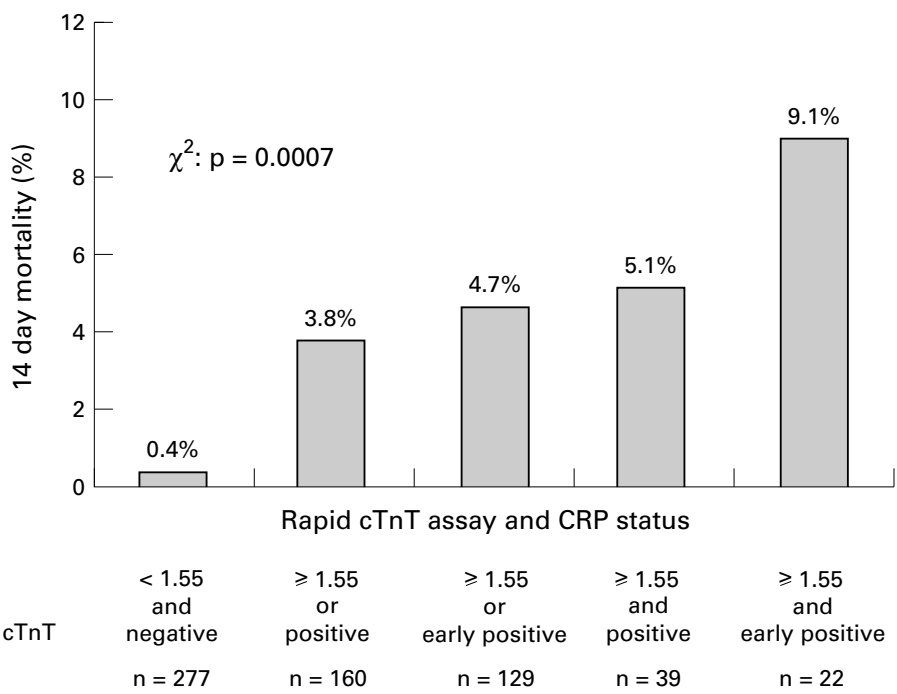

Figure 4 Risk stratification by CRP ( $\mathrm{mg} / \mathrm{dl}$ ) and troponin T rapid assay status expressed as 14 day mortality rate by CRP and rapid cTnT result. Early positive rapid assays are those that could be read in $\leqslant 10$ minutes. The trend was evaluated by $\chi^{2}$ test. Reproduced from Morrow et al ${ }^{12}$ with permission of the American College of Cardiology.

of death in unstable angina can be stratified according to the concentration of cardiac specific troponins $\mathrm{T}$ or I. The troponins may be released into the circulation from myocytes of patients with unstable angina when microemboli are discharged from atherosclerotic plaques. The cardiac specific troponins are undetectable in the serum of healthy persons, and their presence probably indicates the development of small foci of myocardial necrosis secondary to platelet emboli from fragile, unstable plaques. We observed that patients with the highest concentrations of troponin I have a mortality rate almost eight times as high as the patients with the lowest concentrations (fig 3). Data from TIMI $11 \mathrm{~A}$ show how combining the information from two indicators - troponin T and CRP - can provide additional prognostic information (fig 4)..$^{10}$ Patients with a negative troponin $\mathrm{T}$ and low CRP have a very low risk of death. When one indicator is abnormal, the risk rises by a factor of about 10, and when both are abnormal the risk is about 20 times as high as when both are normal. Thus, for patients with negative troponin $\mathrm{T}$ and low CRP, the mortality rate is $0.4 \%$, whereas in patients with high troponin $\mathrm{T}$ and high CRP the mortality rate is $9.1 \%$.

The development of bedside assays that can rapidly measure biochemical markers now makes it possible to identify which causal factors are operating in an individual patient, and direct management accordingly-for example, the concentration of prothrombin frag- ment $1+2$ is an indicator of ongoing thrombosis and fibrinolysis in the circulation. Increased concentrations identified at the time of presentation to hospital will allow antithrombotic treatment to be started without delay. ${ }^{16}$

\section{Conclusion}

Defining the aetiology of unstable angina is likely to become as important in selecting management options in the future as determining the severity and estimating the prognosis are at present. Methods to define the aetiology of unstable angina in individual patients are becoming more widely available. Accurate determination of the cause of this syndrome will allow individualised treatment which may be expected to improve clinical outcome.

1 Braunwald E. Unstable angina: a classification. Circulation 1989;80:410-14.

2 Antman EM, Tanasijevic MJ, Thompson B, et al. Cardiacspecific troponin-I levels to predict risk of mortality in specific troponin-I levels to predict risk of mortality in
patients with acute coronary syndromes. $N$ Engl $f \mathrm{Med}$ 1996;335:1342-9.

3 Maseri A. Pathogenic mechanisms in unstable angina. Heart 1999;82(suppl I):I2-4.

4 Braunwald E. Unstable angina: an etiologic approach to management. Circulation 1998;98:2219-22.

5 Cohen M, Demers C, Gurfinkel EP for the ESSENCE Study Group. A comparison of low-molecular-weight heparin with unfractionated heparin for unstable coronary artery disease. Efficacy and safety of subcutaneous enoxaparin in non-Q-wave coronary events study group. $N$ Engl f Med 1997;337:447-52.

6 Antman E, Braunwald E, McCabe CH, et al. Enoxaparin for the acute and chronic management of unstable angina: results of the TIMI $11 \mathrm{~B}$ trial. Circulation 1998;98:I-504.
EPILOG Investigators. Platelet glycoprotein IIb/III r tor blockade and low-dose heparin during percutaneous tor blockade and low-dose heparin during percutaneous 96.

8 CAPTURE Investigators. Randomised placebo-controlled trial of abciximab before and during coronary intervention in refractory angina: the CAPTURE study. Lancet 1997; 349:1429-34

9 Platelet Receptor Inhibition for Ischaemic Syndrome Management in Patient Limited by Unstable Signs and Symptoms (PRISM-PLUS) Trial Investigators. Inhibition of the platelet glycoprotein IIb/IIIa receptor with tirofiban in unstable angina and non-Q-wave myocardial infarction. N Engl f Med 1998;338:1488-97.

10 Kaski JC, Chester MR, Chen L, et al. Rapid angiographic progression of coronary artery disease in patients with angina pectoris: the role of complex stenosis morphology. Circulation 1995;92:2058-65.

11 Liuzzo G, Biasucci LM, Gallimore R, et al. The prognostic value of C-reactive protein and serum amyloid-A in severe
unstable angina. $N$ Engl 7 Med 1997;331:417-24.

12 Morrow DA, Rifai N, Antman EM, et al. C-reactive protein is a potent predictor of mortality independently and in combination with troponin-T in acute coronary syncombination with troponin- T in acute
dromes. f Am Coll Cardiol 1998;31:1460-5.

13 Kuo CC, Gown AM, Benditt EP, et al. Detection of Chlamydia pneumoniae in aortic lesions of atherosclerosis by immunocytochemical stain. Arterioscler Thromb 1993; 13:1501-4.

14 Gurfinkel E, Bozovich G, Daroca A, et al. Randomised trial of roxithromycin in non-Q-wave coronary syndromes: ROXIS pilot study. ROXIS Study Group. Lancet 1997;350: 404-7.

15 Gupta S, Leatham EW, Carrington E, et al. Chlamydia pneumoniae antibodies, cardiovascular events and azithromycin in male survivors of acute myocardial infarction. Circulation 1997;96:404-7.

16 Merlini PA, Bauer KA, Oltrona L, et al. Persistent activation of the coagulation mechanism in unstable angina and myocardial infarction. Circulation 1994;90:61-8. 